\title{
The efficiency of pulsed-jet propulsion
}

\author{
P. F. LINDEN
}

Department of Applied Mathematics and Theoretical Physics, Centre for Mathematical Sciences, University of Cambridge, Wilberforce Road, Cambridge CB3 0WA, UK

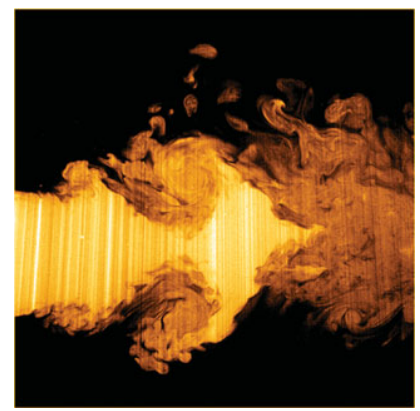

Nature is often an inspiration for scientists and especially so in fluid dynamics. We observe and admire the beauty of birds and fish as they move through the air and water, and wonder how these forms of locomotion evolved and whether they are optimised for efficiency. A common feature of this locomotion is that the thrust is generated by the flapping of a wing or fin, creating an unsteady flow. Other less readily observed animals such as salps or squid eject a pulsed jet, raising the question of whether there is an advantage of this unsteady forcing over a steady jet. This is the question addressed by Ruiz, Whittlesey \& Dabiri (J. Fluid Mech., this issue, vol. 668, 2011, pp. 5-32) who have carried out detailed flow and powerconsumption measurements with a self-propelled vehicle in water. The vehicle has a novel propulsion mechanism that allows a comparison of the efficiency of a pulsed and steady jet to be compared. They show that significant increases in efficiency are possible with the pulsed jet, even allowing for the additional power needed to create the pulsed flow. They also show that vortex rings are produced by the unsteady jet and that the additional entrainment of ambient fluid into the ring and the higher pressure at the front of the ring are the cause of this increased efficiency.

Key words: propulsion, swimming/flying, vortex flows, wakes

\section{Introduction}

Vortex rings have held a fascination for fluid dynamicists for over 150 years. William Barton Rogers, the founder of MIT, conducted experiments and observed that 'the ring is made up of a coil of cloudy air - between the folds of which is rolled a similar coil of transparent atmosphere' (Rogers 1858). In a series of experiments he generated vortex rings by ejection of fluid through a circular orifice, by the rupture of a soap bubble and by dropping one liquid into another liquid. The permanence of vortex rings as fluid dynamical structures was proved by Helmholtz's derivation of the invariant properties of vortex tubes in 1858 (Helmholtz 1867), and this led to Kelvin's provocative 'vortex theory of atoms' (Thompson 1867). Vortex rings have been proposed as paradigm-coherent structures in turbulent flows and are used for underwater drilling and for amusement of dolphins (Shariff \& Leonard 1992).

These early experiments showed two features that are of central interest today in the field of biological locomotion: first, the existence of a vortex ring generated either by the ejection of fluid through an orifice or by flapping of a wing or fin and, second, 
(a)
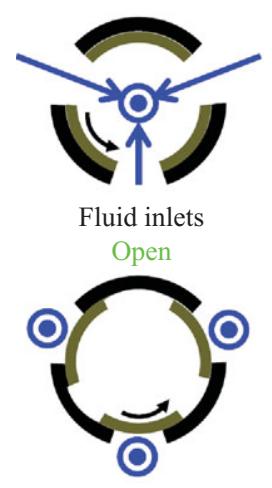

Closed (b)

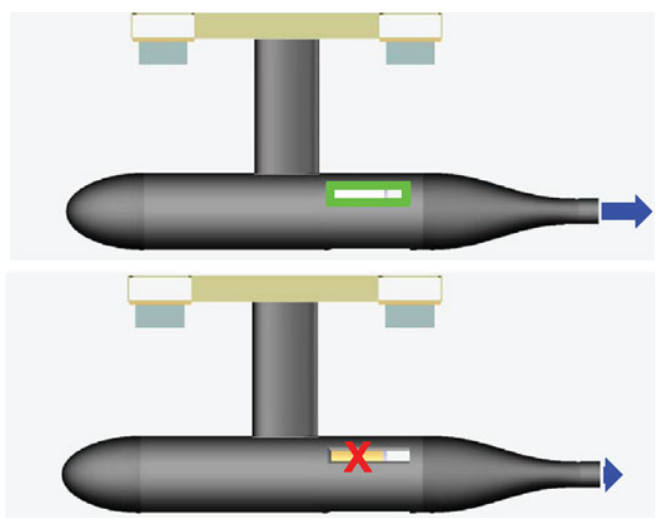

FIGURE 1. Principle of operation for an unsteady, pulsed-jet configuration. (a) View from aft of inlet housing. The outer black arcs indicate inlet housing; the inner grey arcs indicate rotating cylindrical shell. The black arrows indicate the direction of inner shell rotation. The blue arrows indicate flow direction (bull's eyes are vectors out of the page). (b) Side views of the vehicle corresponding to the stages in $(a)$. The green box indicates open inlet; the red cross indicates blocked inlet; the blue arrows at exit indicate the jet efflux magnitude.

the fact that, during its formation, the ring entrains the surrounding fluid. Vortex rings have been clearly identified in the wakes of fish and birds and in animals such as squid and salps that eject fluid rearwards in the form of a limited-duration jet. Since many biological forms of locomotion at high Reynolds numbers involve unsteady, or pulsed, flows it is natural to ask whether these evolved mechanisms are more efficient than steady-propulsion methods.

A major impetus to this question was given by the experiments of Gharib, Rambod \& Shariff (1998) in which they studied the formation of a vortex ring produced by forcing a length $L$ of fluid through an orifice of diameter $D$. They found that there was a critical value of the 'formation number' $L / D$ (about 4 for an injection into a stationary ambient fluid) above which not all the fluid was incorporated into the ring. It was shown that in this limit the impulse of the ring was maximum for a given amount of energy (Linden \& Turner 2001) - in this sense the ring produced at this critical formation number is 'optimal'.

The paper by Ruiz et al. (2011) provides the first experimental verification that pulsed propulsion can be more efficient than propulsion produced by a continuous jet, even allowing for the energy required to generate the pulsed forcing. By making simultaneous measurements of the the flow and the power consumption on a selfpropelled body, Ruiz et al. (2011) confirm recent ideas concerning the role of vortex structures in the wake of a body in increasing the propulsion efficiency.

\section{Overview}

Ruiz et al. (2011) describe an experimental investigation of the flow and forces on a self-propelled vehicle. The vehicle is just over $1 \mathrm{~m}$ long with a maximum diameter of $15.2 \mathrm{~cm}$ and houses its own drive mechanism and method for producing a pulsed jet (figure $1 b$ ). It is attached to a horizontal traverse that provides power and signal 


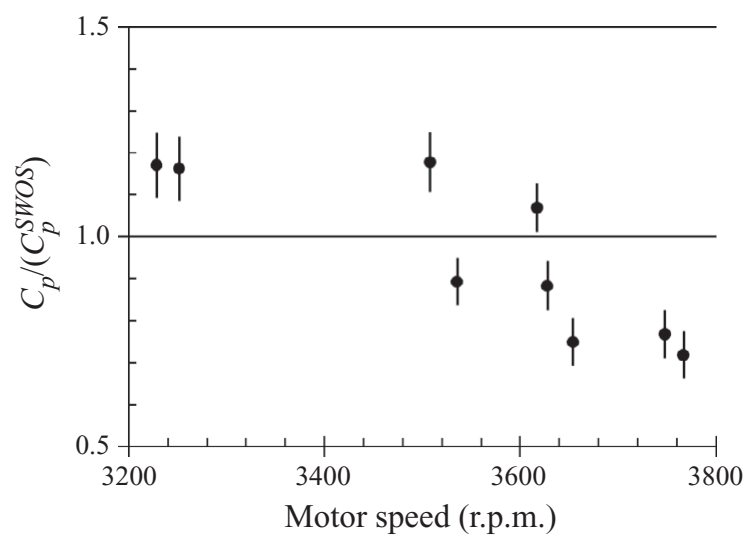

FIGURE 2. Normalised power-consumption coefficient versus motor speed. Normalisations are based on vehicle tests of steady propulsion without a cylindrical rotating shell, denoted by the superscript SWOS.

connections and from which the force can be measured directly. The clever feature of the design is that the mechanism that produces a pulsed jet requires no change in the configuration. This is achieved by providing inlets to the propeller housing that open and close periodically by the rotation of an inner shell (figure $1 a$ ).

Measurements were made of the wake of the stationary vehicle, using digital particle image velocimetry and planar laser-induced fluorescence to obtain, respectively, qualitative and quantitative information about the wake in both the pulsed and steady cases. These showed that the steady propulsor produced a turbulent jet, while in pulsed mode the wake consisted of a sequence of coherent vortex rings (see the figure by the title of the current paper and figures 12 and 13 of Ruiz et al. 2011). Additionally, laser Doppler velocity measurements were made across the wake when the vehicle was moving. These showed that in pulsed mode the wake was wider than the steady jet (figure 14 of Ruiz et al. 2011) as a result of the increased entrainment of ambient fluid by the forming vortex ring as initially discovered by Rogers (1858).

The vehicle's performance was measured directly from the power drawn by the motor driving the propeller and the rotating shell. The key results, (figure 2), are given in terms of a normalised power-consumption coefficient that compares the power consumption of the pulsed propulsor relative to the steady propulsor, including the effects of the rotating shell. At high motor speeds there is a reduction of up to $30 \%$ in the net energy required as a result of the formation of vortex rings.

\section{Future}

These experiments show that a vehicle propelled by a pulsed jet can be significantly more efficient than one propelled by a steady jet. The mechanisms responsible for this increase in efficiency are twofold. The first is the increased entrainment of ambient fluid into the wake during the vortex ring formation. This means that the wake has a lower velocity for the same momentum than in the steady case, resulting in an increase in efficiency. The second mechanism is the downstream acceleration of the vortex ring, including the entrained fluid and the added mass. Kruger \& Gharib 
(2003) have discussed this in terms of an exit overpressure during the ring formation. This increase in pressure occurs because, roughly speaking, the speed at the front of the ring is about one half of the flow entering at the rear. Thus, from Bernoulli considerations, the pressure at the front of the ring is increased compared with that at the exit as the result of this reduction in speed.

This paper, by providing this clear demonstration of increased propulsion efficiency, opens the way for studies to address a range of questions. Do the ideas of single 'optimal' vortices, identified in a number of animal locomotions (e.g Linden \& Turner 2004; Dabiri 2009) carry across to the pulsed-jet case? As Ruiz et al. (2011) point out, they have made no attempt to optimise either the vehicle or the propulsion mechanism. Will the gains in efficiencies measured in their paper also be seen in more optimal vehicle designs? More generally, how do the ideas developed in the paper, such as the effects of vortex ring entrainment and overpressure, apply to vortices generated by the more common flapping mechanisms of propulsion in the animal kingdom?

The paper by Ruiz et al. (2011) also clearly shows the power of imaginatively conceived and carefully executed laboratory experiments. Flow measurements behind swimming fish (e.g. Drucker \& Lauder 1999, 2001) and flying birds (e.g. Hedenstrom $\&$ Spedding 2008) have also revealed that vortices are a common feature of the wake of high-Reynolds-number animal locomotion. Laboratory experiments on vehicles also have the potential to measure the pressure distribution around the vehicle and to address some of the control-volume approximations used in this study. There is also clearly a role for numerical simulations of these flows that will provide diagnostics (e.g. pressure distributions in the wake) that are inaccessible to laboratory experiments.

\section{References}

DABIRI, J. O. 2009 Optimal vortex formation as a unifying principle in biological propulsion. Annu. Rev. Fluid Mech. 41, 17-33.

Drucker, E. G. \& LAUDER, G. V. 1999 Locomotor forces on a swimming fish: three-dimensional vortex wake dynamics quantified using digital particle image velocimetry. J. Exp. Biol. 202, 2393-2412.

Drucker, E. G. \& Lauder, G. V. 2001 Wake dynamics and fluid forces of turning maneuvers in sunfish. J. Expl Biol. 204, 431-442.

Gharib, M., Rambod, E. \& Shariff, K. 1998 A universal timescale for vortex ring formation. J. Fluid Mech. 360, 121-140.

Hedenstrom, A. \& Spedding, G. 2008 Beyond robins: aerodynamic analyses of animal flight. $J$. $R$. Soc. Interface 6, 595-601.

Helmholtz, H. 1867 On integrals of the hydrodynamic equations, which express vortex-motion. Phil. Mag. 33, 485-512.

Kruger, P. S. \& Gharib, M. 2003 The significance of vortex ring formation to the impulse and thrust of a starting jet. Phys. Fluids. 15 (5), 1271-1281.

Linden, P. F. \& Turner, J. S. 2001 The formation of optimal vortex rings and the efficiency of propulsion devices. J. Fluid Mech. 427, 61-72.

Linden, P. F. \& Turner, J. S. 2004 'Optimal' vortex rings and aquatic propulsion. Proc. R. Soc. B 271, 41-63.

RoGERs, W. B. 1858 On the formation of rotating rings of air and liquids under certain conditions of discharge. Am. J. Sci. Arts 26, 247-259.

Ruiz, L. A., Whittlesey, R. W. \& Dabiri, J. O. 2011 Vortex-enhanced propulsion. J. Fluid Mech. 668, 5-32.

Shariff, K. \& Leonard, A. 1992 Vortex rings. Annu. Rev. Fluid Mech. 24, 235-279.

Thompson, W. A. 1867 On vortex atoms. Phil. Mag. 34, 15-24. 